\title{
Reconfigurable Long-Range Phonon Dynamics in Optomechanical Arrays
}

\author{
André Xuereb, ${ }^{1,2, *}$ Claudiu Genes, ${ }^{3,4}$ Guido Pupillo, ${ }^{4}$ Mauro Paternostro, ${ }^{2}$ and Aurélien Dantan ${ }^{5}$ \\ ${ }^{1}$ Department of Physics, University of Malta, Msida MSD 2080, Malta \\ ${ }^{2}$ Centre for Theoretical Atomic, Molecular and Optical Physics, School of Mathematics and Physics, \\ Queen's University Belfast, Belfast BT7 1NN, United Kingdom \\ ${ }^{3}$ Institut für Theoretische Physik, Universität Innsbruck, Technikerstrasse 25, A-6020 Innsbruck, Austria \\ ${ }^{4}$ IPCMS (UMR 7504) and ISIS (UMR 7006), Université de Strasbourg and CNRS, Strasbourg F-67083, France \\ ${ }^{5}$ QUANTOP, Danish National Research Foundation Center for Quantum Optics, Department of Physics and Astronomy, \\ University of Aarhus, 8000 Aarhus C, Denmark
}

(Received 20 December 2013; published 2 April 2014)

\begin{abstract}
We investigate periodic optomechanical arrays as reconfigurable platforms for engineering the coupling between multiple mechanical and electromagnetic modes and for exploring many-body phonon dynamics. Exploiting structural resonances in the coupling between light fields and collective motional modes of the array, we show that tunable effective long-range interactions between mechanical modes can be achieved. This paves the way towards the implementation of controlled phononic walks and heat transfer on densely connected graphs as well as the coherent transfer of excitations between distant elements of optomechanical arrays.
\end{abstract}

DOI: 10.1103/PhysRevLett.112.133604

Introduction.-Optomechanical systems (OMS), naturally lying in the intersection between optical technologies and electronics, play a major role in communication and information-processing sciences [1]. Recent advances in the fabrication of high-quality mechanical resonators and their integration with electromagnetic fields have allowed us to bring the control of mechanical motion to, or close to, the quantum regime, with potential applications in areas as different as metrology and sensing, quantum information processing, and tests of the fundamental laws of physics [2-4]. While these investigations have principally focused on the interplay between electromagnetic radiation and single mechanical resonators, multielement OMS are beginning to be actively studied theoretically [5-18], as well as experimentally [19-24]. The motivations for exploring their potential are manifold. First, their multimode nature makes them well suited for applications in communication technology $[1,10]$. In addition, they hold the promise for enhanced performance in quantum optomechanics and metrology $[14,15]$. Finally, the common interaction of several mechanical elements with one or more electromagnetic fields allows, in principle, for the engineering of complex long-range interactions among the mechanical components, paving the way to the investigation of quantum many-body phenomena with macroscopic elements $[8,14,16,17,25]$. A key challenge in OMS is to engineer reconfigurable systems, in which the interactions are not predetermined by the bulk properties of the system but can be tailored and switched on or off. This would open the way towards, e.g., efficient and controlled manipulation of heat transfer and single excitations in optomechanical arrays.

In this Letter we propose to use periodic optomechanical arrays as reconfigurable platforms for engineering the coupling between multiple mechanical and electromagnetic modes. Such a device operates in a regime where the array
PACS numbers: 42.50.Wk, 07.10.Cm, 07.60.Ly, 42.79.Gn

is transmissive and light permeates through the structure. This allows for both the enhancement of the optomechanical response [14] and the coupling to specific collective motional modes of the array [17]. We show that effective long-range phonon-phonon interactions can be achieved by addressing these transmissive modes. Arising from structural resonances defined by the light fields, these interactions are naturally tunable and reconfigurable. We provide two illustrations of controlled many-body dynamics made possible in this setting. (i) In the bad-cavity regime of optomechanics, the structure acts as a beam splitter array for phonons with effective long-range mode coupling, enabling the investigation of phononic random walks on highly connected graphs and controlled transfer of heat between distant elements in the array; (ii) in the good-cavity regime, coherent and reconfigurable transfer of single excitations is shown to be possible between distant array elements.

These results should enable the investigation of, e.g., nonstandard heat transport and thermodynamics as well as excitation and information transfer in a wide range of periodically ordered OMS, e.g., nanoelectromechanical resonators [26,27], microtoroids [28,29], dielectric membranes [30] or particles [31], optomechanical crystals [32], or cold atoms [24]. The engineering of genuine quantum many-body effects in such an array of mesoscopic systems will provide an additional element into the mechanical quantum simulator that we propose here. This will allow for addressing, e.g., fundamental issues related to the persistence of quantum features in multielement systems with comparatively large masses, dimensions, and at finite temperature. While these conditions would normally imply Newtonian mechanics, the results presented here suggest that clear signatures of nonclassical behavior can persist even in such a mesoscopic simulator. 

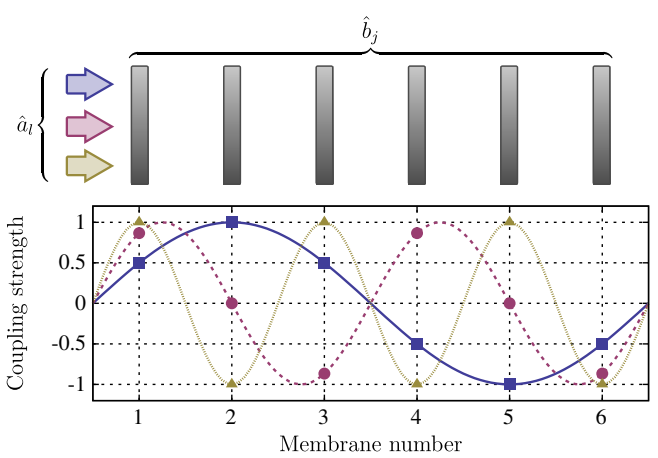

FIG. 1 (color online). Top: Notation used for referring to the mirrors (index $j$ ) and light fields $(l)$. Bottom: Optomechanical coupling for the transmissive modes $l=1$ (squares), $l=2$ (circles), and $l=3$ (triangles) in a six-element array.

Generic model.-We consider the dynamics of an externally driven optomechanical system composed of $N$ identical mechanical elements, here dubbed mirrors, and $N-1$ optical cavity modes. The mirrors are modeled as harmonic oscillators with annihilation operators $\hat{b}_{j}$, vibrational frequency $\omega$, and decay rate $\gamma$. The $l$ th optical mode is detuned by $\Delta_{l}$ with respect to its driving field, has a decay rate $\kappa$, and is described by the annihilation operator $\hat{a}_{l}$. Here, $j=1, \ldots, N$ and $l=1, \ldots, N-1$. We treat the mechanical oscillators as a periodic array of lossless 1D scatterers operating in the Lamb-Dicke regime. Such an array displays $N-1$ optical resonances for which the effective reflectivity vanishes [14,33] and for which the transmissive light modes strongly couple to collective motional modes of the array $[14,17]$. The Hamiltonian of the system reads [34] $(\hbar=1)$

$\hat{H}=\sum_{j, l}\left[\omega \hat{b}_{j}^{\dagger} \hat{b}_{j}+\Delta_{l} \hat{a}_{l}^{\dagger} \hat{a}_{l}+\varepsilon_{l, j}\left(g_{l} \hat{a}_{l}^{\dagger}+g_{l}^{*} \hat{a}_{l}\right)\left(\hat{b}_{j}^{\dagger}+\hat{b}_{j}\right)\right]$,

where the coupling vectors $\mathbf{\epsilon}_{l}=\left(\epsilon_{l, j}\right)_{j}$ are dimensionless, have unit norm, and are determined mainly by the optical properties of the system. In the case of a periodic array of identical scatterers, at the frequencies where the array is transparent these vectors take the sinusoidal form $\epsilon_{l, j} \propto$ $\sin [2 \pi l(j-1 / 2) / N]$ [17]. The optomechanical couplings of the elements thus have a long-ranged sinusoidal profile spanning the whole array (Fig. 1). Each complex frequency $g_{l} \propto \alpha_{l}$ is determined by the mean-field amplitude of the respective mode $\left(\alpha_{l}\right)$ and the overall optomechanical coupling strength multiplying $\mathbf{\epsilon}_{l}$.

Hamiltonian (1) allows for the engineering of a flexible toolbox for the manipulation of phonon dynamics in an optomechanical array. In the following we shall investigate two regimes. (i) In the bad-cavity regime $(\kappa \gg \omega)$, we derive an effective Hamiltonian for the mechanics and investigate phonon diffusion and heat transfer through the array; (ii) in the good-cavity regime $(\kappa \ll \omega)$, we derive an analytical expression for the matrix describing the unitary evolution, which allows for the engineering of controlled coherent phonon dynamics.

Bad-cavity limit. - By generalizing the standard procedure [35], the optical fields can be eliminated from the dynamics
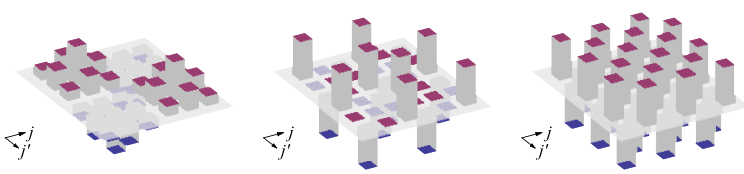

FIG. 2 (color online). Coupling matrices, $\boldsymbol{E}_{l}=\left[\epsilon_{l, j} \epsilon_{l, j^{\prime}}\right]_{j, j^{\prime}}$, which determine how excitations flow throughout the array, for $N=6$. Left to right: $l=1,2,3 ; l=4$ (5) is identical to $l=2$ (1). Positive values in magenta, negative in blue.

of the optomechanical system provided that $\left|g_{l}\right| \ll \omega \ll \kappa$. This yields the effective linear-coupling Hamiltonian $\hat{H}_{\text {eff }}=$ $\sum_{l, j, j^{\prime}} \beta_{l} \epsilon_{l, j} \epsilon_{l, j^{\prime}} \hat{b}_{j}^{\dagger} \hat{b}_{j^{\prime}}$ [34], with $\beta_{l}=2\left|g_{l}\right|^{2} \Delta_{l}\left(\Delta_{l}^{2}-\omega^{2}+\kappa^{2}\right) /$ $\left[\left(\Delta_{l}^{2}-\omega^{2}-\kappa^{2}\right)^{2}+\left(2 \Delta_{l} \kappa\right)^{2}\right]$. The matrices $\boldsymbol{E}_{l}=\left[\epsilon_{l, j} \epsilon_{l, j^{\prime}}\right]_{j, j^{\prime}}$, illustrated in Fig. 2 for $N=6$, and the tuning of $\beta_{l}$, performed by adjusting $g_{l}$ and $\Delta_{l}$, determine how excitations spread through the array. The resulting system is the phononic analog of the random walks explored in Refs. [36-43]. Using the vectors $\boldsymbol{\epsilon}_{l}$ to build an orthonormal similarity matrix $\boldsymbol{P}$, we can cast the evolution of the operators describing the mechanical modes as $\langle\hat{\boldsymbol{b}}(t)\rangle=\left(\boldsymbol{P}^{T} e^{-i \beta t} \boldsymbol{P}\right)\langle\hat{\boldsymbol{b}}(0)\rangle$ [34], where $\boldsymbol{\beta}=$ $\left(\beta_{l} \delta_{l, j}\right)_{l, j}$. In a similar fashion to Ref. [44], the matrix $\boldsymbol{P}$ can be decomposed into linear optics components (cf. Fig. 3), allowing a general and physically transparent description of the dynamics, and illustrating the way phonons flow through the array.

Figure 4 illustrates a situation where phonons are initially prepared in a coherent state localized at one element of the optomechanical array [Fig. 4(a)]. As expected, Fig. 4(b) shows that $\hat{H}_{\text {eff }}$ imposes a final population distribution with a sinusoidal shape mimicking that of $\left(\left|\epsilon_{l, j}\right|^{2}\right)_{j}$. [It can be demonstrated numerically that if the coherences between the different modes are set to zero after each step in the interferometer, the resulting classical distribution does not bear any resemblance to $\left(\left|\epsilon_{l, j}\right|^{2}\right)_{j}$.] Furthermore, we find that the population distribution can be modified by a proper design of the effective beam splitters and phase shifters described above [45]. As examples of this flexibility in the manipulation of phonon dynamics, we impose two kinds of randomness on the system: (i) a random phase offset to the phase shifters making up $\boldsymbol{\beta}$, which can be generated by adding noise to the optical parameters, and (ii) a randomization of the

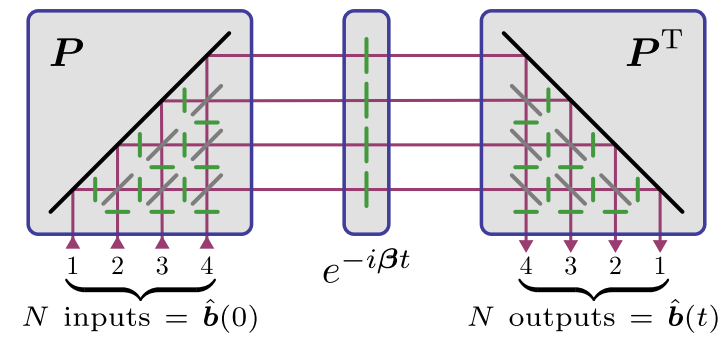

FIG. 3 (color online). Decomposition of the Heisenberg-picture propagator for the vector $\hat{\boldsymbol{b}}$ [44]. $\boldsymbol{P}$ is decomposed into beam splitters (gray, at $45^{\circ}$ ) and phase shifters (green, horizontal and vertical); adding randomness influences the spread of phonons in the array. We show $N=4$. 


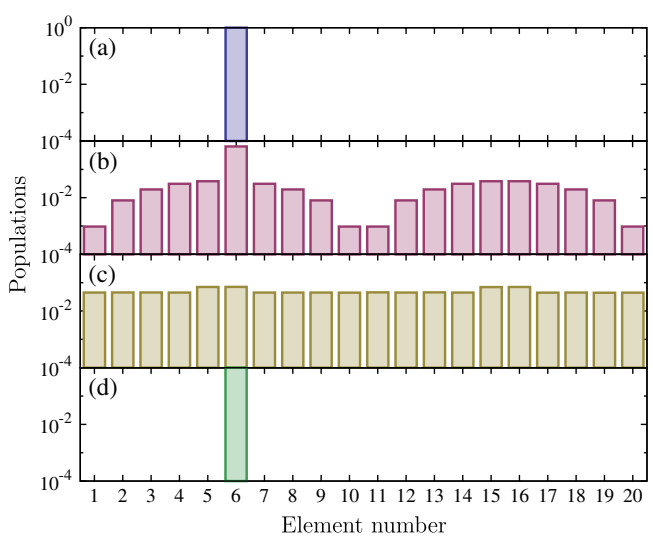

FIG. 4 (color online). A random walk for phonons. Starting from (a) a coherent state launched from the sixth element in a 20-element array, the final populations (b) in the absence of randomness mimic the vector $\mathbf{\epsilon}_{l}$ (we use $l=1$ here). (c) Randomizing the phase shifts in the evolution yields a quasiflat population distribution, whereas (d) randomizing the transmissivity (parametrized as an angle [44]) yields a highly localized distribution. The random angles were drawn from a distribution with mean zero and standard deviation $\pi$; each plot represents an average over 10000 realizations. $\left(\beta_{l>1}=0\right.$, evolution time $t=\pi / \beta_{1} ; \beta_{1} \ll \omega$ is arbitrary.)

transmission of the beam splitters in the decomposition of $\boldsymbol{P}$, which corresponds to perturbing $\epsilon_{l, j}$, i.e., changing the properties of the mechanical elements [17]. In the former case, Fig. 4(c) shows that averaging over many realizations of random phase distributions yields almost uniform phonon populations. In the latter case, Fig. 4(d) shows that disorder has instead the opposite effect: the probability distribution collapses into a highly localized one with significant population only at the element where the excitation was injected. Combinations of these possibilities can be realized, resulting in a flexible control of the type of phonon walk to be implemented.

Let us now explore the flow of heat throughout the array. The Hamiltonian $\hat{H}$ described above is quadratic and therefore preserves the Gaussian nature of any input state of this kind. With this in mind, we constrain the present analysis to the set of Gaussian states. Each of the mechanical elements is coupled to a Markovian bath characterized by a temperature giving rise to a mean number of excitations $\bar{n}_{j}$ in element $j$. We choose $\bar{n}_{j}=\bar{n}+\Delta n \delta_{j, J}$ for $1 \leq J \leq N$. Therefore, each phonon bath has a mean number of excitations $\bar{n}$ except for that of element $J$, which has $\bar{n}+\Delta n$. The heat dynamics in the array is then analyzed by solving the differential equation governing the evolution of the covariance matrix of the $(2 N-1)$ partite system [46].

The adiabatic elimination discussed previously yields a system of $N$ harmonic oscillators coupled not only to the aforementioned $N$ thermal baths, but also to $N-1$ shared reservoirs. These reservoirs, which arise through the coupling of each optical field to collective mechanical modes [17], complicate the picture and prevent the standard identification

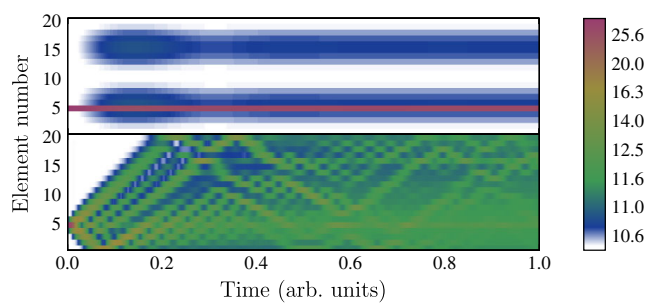

FIG. 5 (color online). Heat diffusion in optomechanical arrays. Top: We evaluate the mean excitation number on each of 20 elements as a function of time; initially each element is in thermal equilibrium with the bath that it is in contact with in the absence of light. The optical field causes excitations to flow from the hotter element to the rest of the array. In marked contrast with a nearest-neighbor coupling (bottom), the flow does not take place via conduction through adjacent elements, but is mediated by the optical field and occurs to the entire array simultaneously. $\left(\gamma / \omega=5 \times 10^{-5}, \kappa / \omega=6.4, \Delta_{l} / \omega=-1, g_{1} / \omega=0.3, g_{>1}=0\right.$, $\bar{n}=10, \Delta n=20$. The nearest-neighbor coupling strength was chosen to be $0.3 \omega$.)

of "heat flowing through an element," in the spirit of Ref. [47]. The alternative we explore in this section is to calculate the occupation number of the $N$ mechanical elements and infer from this the effective heat flow through the array.

The results, illustrated in Fig. 5, exhibit two nonstandard behaviors that are intimately tied with the properties of the optomechanical system under consideration. First, this system exhibits static reconfigurability; i.e., the form of the steady-state phonon population distribution can be chosen by changing which of the optical fields is used to induce the indirect couplings between them. While it is not possible to choose an arbitrary distribution, owing to the symmetry of the effective Hamiltonian, this choice still admits considerable flexibility. Second, the flow of energy from one mechanical element to another takes place indirectly, through the cavity field. It therefore proceeds at a similar rate throughout the entire array, governed not by the distance between the source element $J$ and the element in question but by the coupling constant of the latter to the optical field. A corollary of this is, if $\epsilon_{l, j^{\prime}}=0$ for some $j^{\prime}$, one can speak of heat flow from the element $J<j^{\prime}$ to another element $j>j^{\prime}$ without necessitating any form of heat conduction through element $j^{\prime}$ itself. This situation occurs, e.g., for $l=1$ and $j^{\prime}=(N+1) / 2$ whenever $N$ is odd [17]. For even $N$, the elements closest to the center of the array are the least affected. What distinguishes optically mediated from direct coupling is thus (i) reconfigurability and (ii) time scales, as excitations flow to every element simultaneously in the optical case, rather than sequentially. These studied interactions enable the study of heat transfer and thermodynamics in nonstandard settings $[48,49]$. The parameters selected for plotting Fig. 5 were such that for $\Delta n=0$ the steady-state occupation numbers were, to a good approximation, all equal to $\bar{n}$, regardless of the cooling effects of the optomechanical coupling.

Good-cavity limit.-We now turn to the good-cavity regime, for which $\kappa \ll\left|g_{l}\right| \ll \omega$. This allows us to neglect 


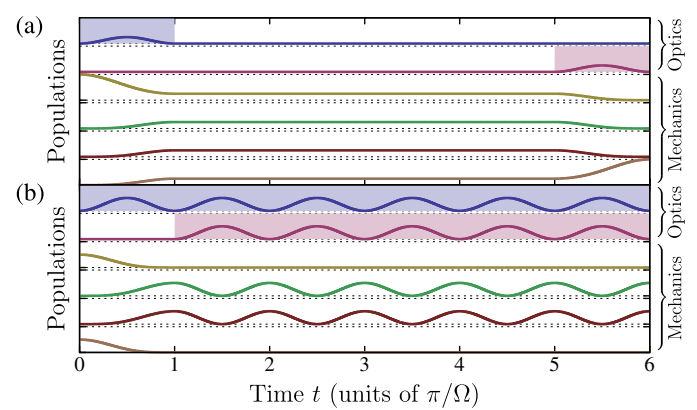

FIG. 6 (color online). Phonon shuttling in an optomechanical array. Shown are (top to bottom) the populations in two optical fields and four mechanical elements, offset for clarity. Shaded regions denote when the mean component of the optical field is nonzero. (a) "Hold-and-switch" protocol: A phonon on element 1 is transferred to a superposition and then to element 4. (b) A phonon initially in a superposition state between elements 1 and 4 is transferred into an excitation shared between the mechanical 2 and 3 and the light fields. For $\omega \gg \kappa$, the short-time dynamics is not affected appreciably upon inclusion of dissipation [34]. ("Off" amplitudes $g_{l}=0$, "on" amplitudes $g_{l}=\Omega ; \Omega \ll \omega$ is arbitrary.)

the nonunitary dynamics in an approximate picture if we confine ourselves to times $\ll 1 / \kappa$. Upon setting $\Delta_{l}=-\omega$, moving into a rotating frame with respect to the free Hamiltonian (i.e., $\hat{H}$ with $g_{l}=0 \forall l$ ), and neglecting rapidly oscillating terms in the Hamiltonian, we obtain the evolution operator

$$
\hat{U}(t)=\exp \left[-i t \sum_{j, l} \epsilon_{l, j}\left(g_{l} \hat{a}_{l}^{\dagger} \hat{b}_{j}+g_{l}^{*} \hat{a}_{l} \hat{b}_{j}^{\dagger}\right)\right] .
$$

Our interest lies in the coherent shuttling of single excitations around the system. We therefore constrain ourselves to the single-excitation subspace and express the state vector as a $(2 N-1)$-dimensional vector with the first $N-1$ (last $N$ ) entries representing the probability amplitude of the excitation to be found in the respective optical (mechanical) mode. To simplify the notation, let us define the matrix $\Lambda=\left[i g_{l}^{*} \epsilon_{l, j}\right]_{l, j}$ ( $N$ columns, $N-1$ rows). It can then be shown that the unitary evolution matrix can be written in the block-matrix form [34]

$$
\boldsymbol{U}(t)=\left[\begin{array}{cc}
\boldsymbol{u}_{11} & \boldsymbol{u}_{12} \\
-\boldsymbol{u}_{12}^{\dagger} & \boldsymbol{u}_{22}
\end{array}\right],
$$

where $\boldsymbol{u}_{11}=\cos \left(t \sqrt{\Lambda \Lambda^{\dagger}}\right), \boldsymbol{u}_{22}=\cos \left(t \sqrt{\Lambda^{\dagger} \Lambda}\right)$, and $\boldsymbol{u}_{12}=$ $-\Lambda \sin \left(t \sqrt{\Lambda^{\dagger} \Lambda}\right)\left(\sqrt{\Lambda^{\dagger} \Lambda}\right)^{-1}[50]$.

In principle, this evolution can even be dynamically reconfigurable if we allow for the possibility that the amplitudes $g_{l}$ of the optical modes can be changed on a time scale $\ll 1 / \omega$, and therefore significantly shorter than any other time scale of the problem. The implementation of this is discussed in detail in Ref. [34]; we note that it is crucial that this switching occurs when the mechanical and optical subsystems are uncorrelated and no excitations reside in the optical subsystem. With this in mind, we can therefore string together sequences of $\boldsymbol{U}(\bullet)$, between which the amplitudes $g_{l}$ are changed instantaneously. The result of this procedure is a set of linear equations that allow us to engineer the route of an excitation through the array. As an example, we illustrate the case for $N=4$, where the fact that $\left|\epsilon_{l, j}\right|=\frac{1}{2}$ independently of $l$ and $j$ allows for particularly simple protocols to be devised. We demonstrate our ideas by means of the two different examples shown in Fig. 6: (a) by switching the amplitudes of two fields, we transport a phonon from mirror 1 to mirror 4, and (b) starting from an initial superposition of the phonon on mirrors 1 and 4 , we end up with a polariton oscillating between mirrors 2 and 3 and the light fields.

Discussion and outlook.-We have investigated collective dynamics in multimode OMS with the goal of simulating many-body effects. The dynamical regimes considered in our analysis showcase distinctive possibilities, ranging from diffusionlike propagation of phononic excitations across the array to the controlled transfer of phonons between targeted elements of the mechanical system. Other regimes of interest could be similarly explored. For instance, operating with blue-detuned cavity fields would allow for investigating collective self-oscillations and synchronization [16] in such systems; exploiting the intrinsic nonlinearity of the optomechanical coupling could enable simulation of many-body models (e.g., the Bose-Hubbard Hamiltonian) [51] or quantum information processing [52] with mechanical systems; and using ring cavities would allow exploring geometric phases [18]. Such studies are promising for engineering nontrivial many-body dynamics, a possibility we plan to pursue in future works addressing dissipative quantum state engineering, dynamical phase transitions, and fluctuation theorems of thermodynamics origin [53-55].

A. X. would like to thank C. Di Franco for interesting discussions and the Royal Commission for the Exhibition of 1851 for financial support. C. G. acknowledges support from the Austrian Science Fund (FWF), P24968-N27, and G.P. from the European Commission through ERC St-Grant "COLDSIM" (No. 307688), AFOSR, and UdS Labex. M.P. thanks the UK EPSRC for a Career Acceleration Fellowship and a grant awarded under the "New Directions for Research Leaders" initiative (EP/ G004579/1), the Alexander von Humboldt Stiftung, the John Templeton Foundation (Grant No. 43467), and the EC Collaborative project TherMiQ (No. 618074). A.D. acknowledges funding from the EU (CCQED project), the Institut Francais du Danemark (IFD2013 program), and the Danish Council for Independent Research (Sapere Aude program). Some calculations were carried out using computational facilities funded by the European Regional Development Fund, Project ERDF-080.

*Corresponding author. andre.xuereb@um.edu.mt

[1] M. Roukes, Phys. World 14, 25 (2001).

[2] T. J. Kippenberg and K. J. Vahala, Science 321, 1172 (2008). 
[3] P. Meystre, Ann. Phys. (Amsterdam) 525, 215 (2013).

[4] M. Aspelmeyer, T. J. Kippenberg, and F. Marquardt, arXiv:1303.0733.

[5] J. Eisert, M. B. Plenio, S. Bose, and J. Hartley, Phys. Rev. Lett. 93, 190402 (2004).

[6] M. Bhattacharya, H. Uys, and P. Meystre, Phys. Rev. A 77, 033819 (2008).

[7] M. J. Hartmann and M. B. Plenio, Phys. Rev. Lett. 101, 200503 (2008).

[8] M. Ludwig, K. Hammerer, and F. Marquardt, Phys. Rev. A 82, 012333 (2010).

[9] J. M. Dobrindt and T. J. Kippenberg, Phys. Rev. Lett. 104, 033901 (2010).

[10] K. Stannigel, P. Rabl, A. S. Sørensen, P. Zoller, and M. D. Lukin, Phys. Rev. Lett. 105, 220501 (2010).

[11] G. Heinrich, M. Ludwig, J. Qian, B. Kubala, and F. Marquardt, Phys. Rev. Lett. 107, 043603 (2011).

[12] D. E. Chang, A. H. Safavi-Naeini, M. Hafezi, and O. Painter, New J. Phys. 13, 023003 (2011).

[13] K. Stannigel, P. Komar, S. J. M. Habraken, S. D. Bennett, M. D. Lukin, P. Zoller, and P. Rabl, Phys. Rev. Lett. 109, 013603 (2012).

[14] A. Xuereb, C. Genes, and A. Dantan, Phys. Rev. Lett. 109, 223601 (2012).

[15] H. Seok, L. F. Buchmann, S. Singh, and P. Meystre, Phys. Rev. A 86, 063829 (2012).

[16] M. Ludwig and F. Marquardt, Phys. Rev. Lett. 111, 073603 (2013).

[17] A. Xuereb, C. Genes, and A. Dantan, Phys. Rev. A 88, 053803 (2013).

[18] S. Chesi, Y.-D. Wang, and J. Twamley, arXiv:1402.0926.

[19] Q. Lin, J. Rosenberg, D. Chang, R. Camacho, M. Eichenfield, K. J. Vahala, and O. Painter, Nat. Photonics 4, 236 (2010).

[20] I. Mahboob, E. Flurin, K. Nishiguchi, A. Fujiwara, and H. Yamaguchi, Nat. Commun. 2, 198 (2011).

[21] I. Mahboob, K. Nishiguchi, H. Okamoto, and H. Yamaguchi, Nat. Phys. 8, 387 (2012).

[22] F. Massel, T. T. Heikkilä, J.-M. Pirkkalainen, S. U. Cho, H. Saloniemi, P. J. Hakonen, and M. A. Sillanpää, Nature (London) 480, 351 (2011).

[23] K. Zhang, P. Meystre, and W. Zhang, Phys. Rev. Lett. 108, 240405 (2012).

[24] T. Botter, D. W. C. Brooks, S. Schreppler, N. Brahms, and D. M. Stamper-Kurn, Phys. Rev. Lett. 110, 153001 (2013).

[25] A. Tomadin, S. Diehl, M. D. Lukin, P. Rabl, and P. Zoller, Phys. Rev. A 86, 033821 (2012).

[26] E. Buks and M. Roukes, J. Microelectromech. Syst. 11, 802 (2002).

[27] J. D. Teufel, T. Donner, D. Li, J. W. Harlow, M. S. Allman, K. Cicak, A. J. Sirois, J. D. Whittaker, K. W. Lehnert, and R. W. Simmonds, Nature (London) 475, 359 (2011).

[28] T. J. Kippenberg, H. Rokhsari, T. Carmon, A. Scherer, and K. J. Vahala, Phys. Rev. Lett. 95, 033901 (2005).

[29] A. Arbabi, Y.M. Kang, C.-Y. Lu, E. Chow, and L. L. Goddard, Appl. Phys. Lett. 99, 091105 (2011).

[30] J. D. Thompson, B. M. Zwickl, A. M. Jayich, F. Marquardt, S. M. Girvin, and J. G. E. Harris, Nature (London) 452, 72 (2008).

[31] O. Romero-Isart, A. C. Pflanzer, M. L. Juan, R. Quidant, N. Kiesel, M. Aspelmeyer, and J. I. Cirac, Phys. Rev. A 83, 013803 (2011).
[32] M. Eichenfield, J. Chan, R. M. Camacho, K. J. Vahala, and O. Painter, Nature (London) 462, 78 (2009).

[33] I. H. Deutsch, R. J. C. Spreeuw, S. L. Rolston, and W. D. Phillips, Phys. Rev. A 52, 1394 (1995).

[34] See Supplemental Material at http://link.aps.org/ supplemental/10.1103/PhysRevLett.112.133604, which includes Ref. [57-73], for more details and the intermediate steps of our calculations.

[35] C. W. Gardiner, Phys. Rev. A 29, 2814 (1984).

[36] M. A. Broome, A. Fedrizzi, B. P. Lanyon, I. Kassal, A. Aspuru-Guzik, and A. G. White, Phys. Rev. Lett. 104, 153602 (2010).

[37] A. Peruzzo, A. Laing, A. Politi, T. Rudolph, and J. L. O’Brien, Nat. Commun. 2, 224 (2011).

[38] A. Schreiber, K. N. Cassemiro, V. Potoček, A. Gábris, I. Jex, and C. Silberhorn, Phys. Rev. Lett. 106, 180403 (2011).

[39] L. Sansoni, F. Sciarrino, G. Vallone, P. Mataloni, A. Crespi, R. Ramponi, and R. Osellame, Phys. Rev. Lett. 108, 010502 (2012).

[40] M. Tillmann, B. Dakić, R. Heilmann, S. Nolte, A. Szameit, and P. Walther, Nat. Photonics 7, 540 (2013).

[41] J. B. Spring, B. J. Metcalf, P. C. Humphreys, W. S. Kolthammer, X.-M. Jin, M. Barbieri, A. Datta, N. ThomasPeter, N. K. Langford, D. Kundys, J. C. Gates, B. J. Smith, P. G. R. Smith, and I. A. Walmsley, Science 339, 798 (2013).

[42] A. Crespi, R. Osellame, R. Ramponi, V. Giovannetti, R. Fazio, L. Sansoni, F. De Nicola, F. Sciarrino, and P. Mataloni, Nat. Photonics 7, 322 (2013).

[43] Y.-C. Jeong, C. Di Franco, H.-T. Lim, M. S. Kim, and Y.-H. Kim, Nat. Commun. 4, 2471 (2013).

[44] M. Reck, A. Zeilinger, H. J. Bernstein, and P. Bertani, Phys. Rev. Lett. 73, 58 (1994).

[45] H. Jeong, M. Paternostro, and M. S. Kim, Phys. Rev. A 69, 012310 (2004).

[46] A. Ferraro, S. Olivares, and M. G. A. Paris, Gaussian States in Quantum Information, Napoli Series on Physics and Astrophysics (Bibliopolis, Napoli, 2005).

[47] A. Asadian, D. Manzano, M. Tiersch, and H.-J. Briegel, Phys. Rev. E 87, 012109 (2013).

[48] Y. Dubi and M. Di Ventra, Rev. Mod. Phys. 83, 131 (2011).

[49] A. Bermudez, M. Bruderer, and M. B. Plenio, Phys. Rev. Lett. 111, 040601 (2013).

[50] This is a multimode generalization, restricted to the single-excitation subspace, of the Jaynes-Cummings evolution operator [56].

[51] K. Jacobs, arXiv:1209.2499.

[52] S. Rips and M. J. Hartmann, Phys. Rev. Lett. 110, 120503 (2013).

[53] L. Mazzola, G. De Chiara, and M. Paternostro, Phys. Rev. Lett. 110, 230602 (2013).

[54] R. Dorner, S. R. Clark, L. Heaney, R. Fazio, J. Goold, and V. Vedral, Phys. Rev. Lett. 110, 230601 (2013).

[55] L. Mazzola, G. De Chiara, and M. Paternostro, arXiv:1401.0566.

[56] S. Stenholm, Phys. Rep. 6, 1 (1973).

[57] K. Jaehne, K. Hammerer, and M. Wallquist, New J. Phys. 10, 095019 (2008).

[58] C. W. Gardiner and P. Zoller, Quantum Noise (Springer, New York, 2004) 3rd ed.

[59] C. K. Law, Phys. Rev. A 51, 2537 (1995). 
[60] M. Karuza, C. Biancofiore, M. Bawaj, C. Molinelli, M. Galassi, R. Natali, P. Tombesi, G. Di Giuseppe, and D. Vitali, Phys. Rev. A 88, 013804 (2013).

[61] Q. Xu, P. Dong, and M. Lipson, Nat. Phys. 3, 406 (2007).

[62] K. Kondo, M. Shinkawa, Y. Hamachi, Y. Saito, Y. Arita, and T. Baba, Phys. Rev. Lett. 110, 053902 (2013).

[63] Y.-C. Liu, Y.-F. Xiao, X. Luan, and C. W. Wong, Phys. Rev. Lett. 110, 153606 (2013).

[64] F. Massel, S. U. Cho, J.-M. Pirkkalainen, P. J. Hakonen, T. T. Heikkilä, and M. A. Sillanpää, Nat. Commun. 3, 987 (2012).

[65] A. D. O'Connell, M. Hofheinz, M. Ansmann, R. C. Bialczak, M. Lenander, E. Lucero, M. Neeley, D. Sank, H. Wang, M. Weides, J. Wenner, J. M. Martinis, and A. N. Cleland, Nature (London) 464, 697 (2010).

[66] J.-M. Pirkkalainen, S. U. Cho, J. Li, G. S. Paraoanu, P. J. Hakonen, and M. A. Sillanpää, Nature (London) 494, 211 (2013).
[67] T. P. Purdy, R. W. Peterson, and C. A. Regal, Science 339, 801 (2013).

[68] S. Lee, V. P. Adiga, R. A. Barton, A. M. van der Zande, G.-H. Lee, B. R. Ilic, A. Gondarenko, J. M. Parpia, H. G. Craighead, and J. Hone, Nano Lett. 13, 4275 (2013).

[69] V. P. Adiga, R. De Alba, I. R. Storch, P. A. Yu, B. Ilic, R. A. Barton, S. Lee, J. Hone, P. L. McEuen, J. M. Parpia, and H. G. Craighead, Appl. Phys. Lett. 103, 143103 (2013).

[70] T. Bagci, A. Simonsen, S. Schmid, L. G. Villanueva, E. Zeuthen, J. Appel, J. M. Taylor, A. Sørensen, K. Usami, A. Schliesser, and E. S. Polzik, arXiv:1307.3467.

[71] R. W. Andrews, R. W. Peterson, T. P. Purdy, K. Cicak, R. W. Simmonds, C. A. Regal, and K. W. Lehnert, arXiv:1310.5276.

[72] D. M. Stamper-Kurn, arXiv:1204.4351.

[73] N. Brahms, T. Botter, S. Schreppler, D. W. C. Brooks, and D. M. Stamper-Kurn, Phys. Rev. Lett. 108, 133601 (2012). 